\title{
Some moment inequalities for fuzzy martingales and their applications
}

\author{
Hamed Ahmadzade ${ }^{1}$, Mohammad Amini $^{1 *}$, Seyed Mahmoud Taheri ${ }^{2}$ and Abolghasem Bozorgnia ${ }^{1}$
}

\author{
${ }^{*}$ Correspondence: \\ m-amini@um.ac.ir \\ ${ }^{1}$ Department of Statistics, Faculty of \\ Mathematical Sciences, Ferdows \\ University of Mashhad, Mashhad \\ 91775-1159, Iran \\ Full list of author information is \\ available at the end of the article
}

\begin{abstract}
Martingales are a class of stochastic processes which has had profound influence on the development of probability theory and stochastic processes. Some recent developments are related to mathematical finance. In the real world, some information about these phenomena might be imprecise and represented in the form of vague quantities. In these situations, we need to generalize classical methods to vague environment. Thus, fuzzy martingales have been extended as a vague perception of real-valued martingales. In this paper, some moment inequalities are presented for fuzzy martingales. Several convergence theorems are established based on these inequalities. As an application of convergence theorems, a weak law of large numbers for fuzzy martingales is stated. Furthermore, a few examples are devoted to clarify the main results.
\end{abstract}

Keywords: Fuzzy random variable; Fuzzy martingale; Weak convergence; Strong convergence

\section{Introduction}

Over the last decades, the theory of fuzzy random variables has been extensively developed. A fuzzy random variable has been extended as a vague perception of a real-valued random variable and subsequently redefined as a particular random set, see e.g., [1-5]. We review several researches on this topic. A strong law of large numbers for fuzzy random variables was given by Miyakoshi and Shimbo [2]. Klement et al. [6] established a strong law of large numbers for fuzzy random variables, based on embedding theorem as well as certain probability techniques in the Banach spaces. Taylor et al. [7] proved a weak law of large numbers for fuzzy random variables in separable Banach spaces. Joo et al. [8] established Chung-type strong law of large numbers for fuzzy random variables based on isomorphic isometric embedding theorem. Fu and Zhang [9] obtained some strong limit theorems for fuzzy random variables with slowly varying weight. It should be mentioned that although the concept of variance has been found very convenient in studying limit theorems, but, as the authors know, the limit theorems for fuzzy random variables based on the concept of variance has not been developed, except in the works by Korner [10] and Feng [11]. Korner [10] proved the strong and weak laws of large numbers for fuzzy random variables. Based on a natural extension of the concept of variance, he extended Kolmogorov's inequality to independent fuzzy random variables and derived several limit theorems. Their methods are direct applications of classical methods in probability theory

(C) 2014 Ahmadzade et al.; licensee Springer. This is an Open Access article distributed under the terms of the Creative Commons Attribution License (http://creativecommons.org/licenses/by/2.0), which permits unrestricted use, distribution, and reproduction in any medium, provided the original work is properly cited. 
to fuzzy random variables. In this paper, some moment inequalities for fuzzy martingales are established and convergence theorems of such fuzzy random variables are studied. We survey some topics in real-valued martingale theory and fuzzy martingale theory. The term martingale originates from the gambling theory. The famous gambling strategy to double one's stake as long as one loses and leave as soon as one wins is called a martingale. Now, if a gambling value is vague with a certain membership function, the pervious strategy is called fuzzy martingale. A classic interpretation of martingales in the context of gambling is given as follows. Let $X_{n}$ represent the fortune of a gambler at the end of the $n$th play, and let $\mathcal{F}_{n}$ be the information available to the gambler up to and including the $n$th play. Then, $\mathcal{F}_{n}$ contains the knowledge of all events like $\left\{X_{j} \leq r\right.$, for $\left.r \in \mathbb{R}, j \leq n\right\}$ making $X_{n}$ measurable with respect to $\mathcal{F}_{n}$. The mathematical definition of martingale $\left(E\left(X_{n+1} \mid \mathcal{F}_{n}\right)=X_{n}\right)$ says that the given all the information up until the end of the $n$th play, the expected fortune of the gambler at the end of the $(n+1)$ th play remains unchanged. Thus, a martingale represents a fair game. In situation where the game puts the gambler in a favorable position, one may express sub-martingale i.e., $\left(E\left(X_{n+1} \mid \mathcal{F}_{n}\right) \geq X_{n}\right)$. It is mentioned that limit theorems play important roles in studying some practical instances of martingale theory such as extinction in branching process and bankruptcy in investment problems like portfolio selection.

The concept of fuzzy martingale and its properties is introduced and studied by Puri and Ralescu [12], Stojakovic [13], and Feng [14]. Puri and Ralescu [12] proved some convergence theorems of martingale. Stojakovic [13] discussed the properties of martingales. Feng [14] studied fuzzy conditional expectation and fuzzy martingale and investigated some results on convergence of fuzzy martingales. Fei et al. [15] and Fei [16] obtained several results on stopping theorem for fuzzy (super-, sub-) martingales with discrete time.

The rest of this paper is organized as follows. In Section 'Preliminaries', we briefly review some preliminary concepts of fuzzy arithmetic and fuzzy random variables. In Section 'Moment Inequalities', several useful inequalities are provided for fuzzy martingales. In Section 'Convergence Theorems', some limit theorems are established to fuzzy martingales. The conclusions are discussed in Section 'Conclusions'.

\section{Preliminaries}

In this section, we provide several definitions and elementary concepts of fuzzy arithmetic and fuzzy random variables that will be used in the next sections. For more details, the reader is referred to $[14,17,18]$.

Define $F(\mathbb{R})=\{\tilde{u}: \mathbb{R} \rightarrow[0,1] \mid \tilde{u}$ satisfies i to iii $\}$; where (i) $\tilde{u}$ is normal, (ii) $\tilde{u}$ is fuzzy convex, and (iii) $\tilde{u}$ is upper semicontinuous. Any $\tilde{u} \in F(\mathbb{R})$ is called a fuzzy number.

For a $\tilde{u} \in F(\mathbb{R}),[\tilde{u}]^{r}=\{x \in \mathbb{R} \mid \tilde{u}(x) \geq r, 0<r \leq 1\}$ is called the r-level set of $\tilde{u}$.

We use the notations $\oplus, \ominus$, and $\odot$, which are defined as follows in this paper:

(i) $[\tilde{a} \oplus \tilde{b}]^{r}=\left[\tilde{a}^{-}(r)+\tilde{b}^{-}(r), \tilde{a}^{+}(r)+\tilde{b}^{+}(r)\right]$.

(ii) If $\lambda>0$, then $[\lambda \odot \tilde{a}]^{r}=\left[\lambda \tilde{a}^{-}(r), \lambda \tilde{a}^{+}(r)\right]$.

(iii) If $\lambda<0$, then $[\lambda \odot \tilde{a}]^{r}=\left[\lambda \tilde{a}^{+}(r), \lambda \tilde{a}^{-}(r)\right]$.

(iv) $[\tilde{a} \ominus \tilde{b}]^{r}=\left[\tilde{a}^{-}(r)-\tilde{b}^{+}(r), \tilde{a}^{+}(r)-\tilde{b}^{-}(r)\right]$.

Let $\tilde{u}, \tilde{v} \in F(\mathbb{R})$, and set $d_{p}(\tilde{u}, \tilde{v})=\left(\int_{0}^{1} h^{p}\left([\tilde{u}]^{r},[\tilde{v}]^{r}\right) d r\right)^{\frac{1}{p}}, 1 \leq p<\infty$, $d_{\infty}(\tilde{u}, \tilde{v})=\sup _{0<r \leq 1} h\left([\tilde{u}]^{r},[\tilde{v}]^{r}\right)$, where $h$ is the Hausdorff metric, i.e., $h\left([\tilde{u}]^{r},[\tilde{v}]^{r}\right)=$ $\max \left\{\left|u^{-}(r)-v^{-}(r)\right|,\left|u^{+}(r)-v^{+}(r)\right|\right\}$. Norm $\|\tilde{u}\|_{p}$ of a fuzzy number $\tilde{u} \in F(\mathbb{R})$ is defined 
by $\|\tilde{u}\|_{p}=d_{p}(\tilde{u}, \tilde{0})$, where $\tilde{0}$ is the fuzzy number in $F(\mathbb{R})$ whose membership function equals 1 at 0 and zero otherwise. The norm $\|\cdot\|_{\infty}$ of $\tilde{u}$ is defined by $\|\tilde{u}\|_{\infty}=d_{\infty}(\tilde{u}, \tilde{0})$. The operation $\langle.,\rangle:. F(\mathbb{R}) \times F(\mathbb{R}) \rightarrow[-\infty, \infty]$ is defined by $\langle\tilde{u}, \tilde{v}\rangle=\int_{0}^{1}\left(\tilde{u}^{-}(r) \tilde{v}^{-}(r)+\right.$ $\left.\tilde{u}^{+}(r) \tilde{v}^{+}(r)\right) d r$. If the indeterminacy of the form $\infty-\infty$ arises in the Lebesgue integral, then we say that $\langle\tilde{u}, \tilde{v}\rangle$ does not exist. It is easy to see that the operation $\langle.,$.$\rangle has the$ following properties:

(i) $\langle\tilde{u}, \tilde{u}\rangle \geq 0$ and $\langle\tilde{u}, \tilde{u}\rangle=0 \Leftrightarrow \tilde{u}=\tilde{0}$.

(ii) $\langle\tilde{u}, \tilde{v}\rangle=\langle\tilde{v}, \tilde{u}\rangle$.

(iii) $\langle\tilde{u}+\tilde{v}, \tilde{w}\rangle=\langle\tilde{u}, \tilde{w}\rangle+\langle\tilde{v}, \tilde{w}\rangle$.

(iv) $\langle\lambda \tilde{u}, \tilde{v}\rangle=\lambda\langle\tilde{u}, \tilde{v}\rangle$.

(v) $|\langle\tilde{u}, \tilde{v}\rangle|<\sqrt{\langle\tilde{u}, \tilde{u}\rangle\langle\tilde{v}, \tilde{v}\rangle}$.

For all $\tilde{u}, \tilde{v} \in F(\mathbb{R})$, if $\langle\tilde{u}, \tilde{u}\rangle<\infty$ and $\langle\tilde{v}, \tilde{v}\rangle<\infty$, then the property (v) implies that $\langle\tilde{u}, \tilde{v}\rangle<\infty$. So, we can define $d_{*}(\tilde{u}, \tilde{v})=\sqrt{\langle\tilde{u}, \tilde{u}\rangle-2\langle\tilde{u}, \tilde{v}\rangle+\langle\tilde{v}, \tilde{v}\rangle}$. In fact, $d_{*}$ is a metric in $\{\tilde{u} \in F(\mathbb{R}) \mid\langle\tilde{u}, \tilde{u}\rangle<\infty\}$.

Moreover, the norm $\|\tilde{u}\|_{*}$ of fuzzy number $\tilde{u} \in F(\mathbb{R})$ is defined by $\|\tilde{u}\|_{*}=d_{*}(\tilde{u}, \tilde{0})$.

Let $(\Omega, \mathcal{A}, P)$ be a probability space. A fuzzy random variable (briefly, FRV) is a Borel measurable function $\tilde{X}:(\Omega, \mathcal{A}) \rightarrow\left(F(\mathbb{R}), d_{\infty}\right)[14]$. Let $\tilde{X}$ be a FRV defined on $(\Omega, \mathcal{A}, P)$. Then $[\tilde{X}]^{r}=\left[\tilde{X}^{-}(r), \tilde{X}^{+}(r)\right], r \in(0,1]$, is a random closed interval, and $\tilde{X}^{-}(r)$ and $\tilde{X}^{+}(r)$ are real-valued random variables. A FRV $\tilde{X}$ is called integrably bounded if $E\|\tilde{X}\|_{\infty}<\infty$, and the expectation value $E \tilde{X}$ is defined as the unique fuzzy number which satisfies the property $[E \tilde{X}]^{r}=E[\tilde{X}]^{r}, 0<r \leq 1[3]$.

Definition 1. ([14]) Let $\tilde{X}$ and $\tilde{Y}$ be two FRVs in $L_{2}\left(L_{2}=\left\{\tilde{X} \mid \tilde{X}\right.\right.$ is FRV and $E\|\tilde{X}\|_{2}^{2}<$ $\infty\})$, then $\operatorname{Cov}(\tilde{X}, \tilde{Y})=\frac{1}{2} \int_{0}^{1}\left(\operatorname{Cov}\left(\tilde{X}^{-}(r), \tilde{Y}^{-}(r)\right)+\operatorname{Cov}\left(\tilde{X}^{+}(r), \tilde{Y}^{+}(r)\right)\right) d r$. Specially, the variance of $\tilde{X}$ is defined by $\operatorname{Var}(\tilde{X})=\operatorname{Cov}(\tilde{X}, \tilde{X})$.

Theorem 1. ([14]) Let $\tilde{X}$ and $\tilde{Y}$ be two FRVs in $L_{2}$ and $\tilde{u}, \tilde{v} \in F(\mathbb{R})$ and $\lambda, k \in \mathbb{R}$. Then

(i) $\operatorname{Cov}(\tilde{X}, \tilde{Y})=\frac{1}{2}(E\langle\tilde{X}, \tilde{Y}\rangle-\langle E \tilde{X}, E \tilde{Y}\rangle)$,

(ii) $\operatorname{Var}(\tilde{X})=\frac{1}{2} E d_{*}^{2}(\tilde{X}, E \tilde{X})$,

(iii) $\operatorname{Cov}(\lambda \tilde{X} \oplus \tilde{u}, k \tilde{Y} \oplus \tilde{v})=\lambda k \operatorname{Cov}(\tilde{X}, \tilde{Y})$,

(iv) $\operatorname{Var}(\lambda \tilde{X} \oplus u)=\lambda^{2} \operatorname{Var}(\tilde{X})$,

(v) $\operatorname{Var}(\tilde{X} \oplus \tilde{Y})=\operatorname{Var}(\tilde{X})+\operatorname{Var}(\tilde{Y})+2 \operatorname{Cov}(\tilde{X}, \tilde{Y})$.

Definition 2. ([19]) The $D_{p, q}$ distance, indexed by parameters $1 \leq p<\infty, 0 \leq q \leq 1$, between two fuzzy numbers $\tilde{u}$ and $\tilde{v}$ is a nonnegative function on $F(\mathbb{R}) \times F(\mathbb{R})$ given as follows: $D_{p, q}(\tilde{u}, \tilde{v})=\left[(1-q) \int_{0}^{1}\left|\tilde{u}^{-}(r)-\tilde{v}^{-}(r)\right|^{p} d r+q \int_{0}^{1}\left|\tilde{u}^{+}(r)-\tilde{v}^{+}(r)\right|^{p} d r\right]^{\frac{1}{p}}$.

Remark 1. If $p=2, q=\frac{1}{2}$, then the metric $D_{p, q}$ is equal to the metric $d_{*}$; for more details, see [16].

To prove the main results, we need to apply an order relation. Thus, we use notations $\prec, \succ, \preceq$, and $\succeq$ defined as follows [18]:

(i) $\tilde{a} \prec \tilde{b}$ if and only if $a^{-}(r)<b^{-}(r)$ and $a^{+}(r)<b^{+}(r) \forall r \in[0,1]$.

(ii) $\tilde{a} \succ \tilde{b}$ if and only if $a^{-}(r)>b^{-}(r)$ and $a^{+}(r)>b^{+}(r) \forall r \in[0,1]$. 
(iii) $\quad \tilde{a} \preceq \tilde{b}$ if and only if $a^{-}(r) \leq b^{-}(r)$ and $a^{+}(r) \leq b^{+}(r) \forall r \in[0,1]$.

(iv) $\tilde{a} \geq \tilde{b}$ if and only if $a^{-}(r) \geq b^{-}(r)$ and $a^{+}(r) \geq b^{+}(r) \forall r \in[0,1]$.

Definition 3. ([20]) Two fuzzy random variables $\tilde{X}$ and $\tilde{Y}$ are called independent if two $\sigma$-fields $\sigma(\tilde{X})=\sigma\left(\left\{X^{-}(r), X^{+}(r) \mid r \in[0,1]\right\}\right)$ and $\sigma(\tilde{Y})=\sigma\left(\left\{Y^{-}(r), Y^{+}(r) \mid r \in[0,1]\right\}\right)$ are independent.

Definition 4. A finite collection of FRVs $\left\{\tilde{X}_{k}, 1 \leq k \leq n\right\}$ is said to be independent if $\sigma$-fields $\sigma\left(\left\{\tilde{X}_{k}^{-}(r), \tilde{X}_{k}^{+}(r) \mid r \in[0,1]\right\} \mid 1 \leq k \leq n\right)$ are independent. An infinite sequence $\left\{\tilde{X}_{n}, n \geq 1\right\}$ is called independent if every finite sub-collection of it is independent.

Definition 5. ([15]) A fuzzy conditional expectation of $\tilde{X}$ with respect to the sub- $\sigma$ field $\mathcal{B}$ of $\mathcal{A}$, denoted as $E(\tilde{X} \mid \mathcal{B})$, is defined as a FRV which satisfies in the following conditions:

(i) $\quad E(\tilde{X} \mid \mathcal{B})$ is $\mathcal{B}$ - measurable.

(ii) $\int_{B} E(\tilde{X} \mid \mathcal{B}) d P=\int_{B} \tilde{X} d P$ for every $B \in \mathcal{B}$. Note that $\int_{B} \tilde{X} d P$ is the Aumann integral of the FRV $\tilde{X}[21]$.

Proposition 1. ([15]) The fuzzy conditional expectation has the following properties:

(i) $\quad E(a \odot \tilde{X} \oplus b \odot \tilde{Y} \mid \mathcal{B})=(a \odot E(\tilde{X} \mid \mathcal{B})) \oplus(b \odot E(\tilde{Y} \mid \mathcal{B}))$ a.s.

(ii) $\tilde{X}$ is $\mathcal{B}$-measurable, then $E(\tilde{X} \mid \mathcal{B})=\tilde{X}$ a.s.

(iii) $E E(\tilde{X} \mid \mathcal{B})=E \tilde{X}$.

(iv) If $\tilde{X} \preceq \tilde{Y}$ a.s. then $E(\tilde{X} \mid \mathcal{B}) \preceq E(\tilde{Y} \mid \mathcal{B})$ a.s.

(v) $\quad d_{\infty}(E(\tilde{X} \mid \mathcal{B}), E(\tilde{Y} \mid \mathcal{B})) \leq E\left(d_{\infty}(\tilde{X}, \tilde{Y}) \mid \mathcal{B}\right)$ a.s. and consequently

$$
\|E(\tilde{X} \mid \mathcal{B})\|_{\infty} \leq E\left(\|\tilde{X}\|_{\infty} \mid \mathcal{B}\right) \text { a.s. }
$$

Definition 6. ([15]) The sequence $\left\{\tilde{X}_{n}, \mathcal{B}_{n}\right\}$ of fuzzy random variables and $\sigma$-algebras is called a fuzzy martingale if we have, for each $n \geq 1$ :

(a) $\tilde{X}_{n}$ is $\mathcal{B}_{n}$-measurable and $E\left\|\tilde{X}_{n}\right\|_{\infty}<\infty$.

(b) $\quad E\left(\tilde{X}_{n+1} \mid \mathcal{B}_{n}\right)=\tilde{X}_{n}$.

The sequence $\left\{\tilde{X}_{n}, \mathcal{B}_{n}\right\}$ is called a fuzzy sub-martingale if property (b) is replaced by

(b') $\quad E\left(\tilde{X}_{n+1} \mid \mathcal{B}_{n}\right) \succeq \tilde{X}_{n}$.

For more on fuzzy martingale and related topics, see e.g. [11,15,22].

Definition 7. ([20]) Let $\tilde{X}$ and $\tilde{X}_{n}$ be FRVs defined on the same probability space $(\Omega, \mathcal{A}, P)$.

(i) We say that $\left\{\tilde{X}_{n}\right\}$ converges to $\tilde{X}$ in probability with respect to the metric $d$ if

$$
\lim _{n \rightarrow \infty} P\left(\omega: d\left(\tilde{X}_{n}(\omega), \tilde{X}(\omega)\right)>\epsilon\right)=0, \quad \forall \epsilon>0 .
$$

(ii) We say that $\left\{\tilde{X}_{n}\right\}$ converges to $\tilde{X}$ almost surely (briefly, a.s.) with respect to the metric $d$, if

$$
P\left(\omega: \lim _{n \rightarrow \infty} d\left(\tilde{X}_{n}(\omega), \tilde{X}(\omega)\right)=0\right)=1 .
$$


(iii) We say that $\left\{\tilde{X}_{n}\right\}$ converges to $\tilde{X}$ in $L^{2}$ with respect to the metric $d$, if

$$
E^{\frac{1}{2}} d^{2}\left(\tilde{X}_{n}, \tilde{X}\right) \rightarrow 0, \text { as } n \rightarrow \infty
$$

It is mentioned that in the literature of probability theory, convergence in probability and almost surely convergence are called weak and strong convergence, respectively.

Throughout the paper, $\tilde{S}_{n}=\oplus_{i=1}^{n} \tilde{X}_{i}$ and $\mathcal{F}_{n}=\sigma\left(\tilde{X}_{1}, \ldots, \tilde{X}_{n}\right)$.

\section{Moment Inequalities}

In this section, we prove some basic inequalities for fuzzy martingales.

The following lemma is essential to obtain our main results.

Lemma 1. If $\left\{\tilde{X}_{n}, n \geq 1\right\}$ is a sequence of FRVs such that $E\left(\tilde{X}_{n} \mid \mathcal{F}_{n-1}\right)=\tilde{0}$, for all $n \geq 1$, then $\left\{\tilde{S}_{n}, \mathcal{F}_{n}\right\}$ is a fuzzy martingale.

Proof. By using linearity property of conditional fuzzy expectation, we obtain

$$
E\left(\tilde{S}_{n} \mid \mathcal{F}_{n-1}\right)=E\left(\tilde{S}_{n-1} \mid \mathcal{F}_{n-1}\right) \oplus E\left(\tilde{X}_{n} \mid \mathcal{F}_{n-1}\right)
$$

Since $\tilde{S}_{n-1}$ is $\mathcal{F}_{n-1}$ measurable and $E\left(\tilde{X}_{n} \mid \mathcal{F}_{n-1}\right)=\tilde{0} \forall n \geq 1$, we have

$$
E\left(\tilde{S}_{n} \mid \mathcal{F}_{n-1}\right)=\tilde{S}_{n-1}
$$

This completes the proof.

Corollary 1. If $\{\tilde{X}, n \geq 1\}$ is a sequence of independent FRVs such that $E\left(\tilde{X}_{n}\right)=\tilde{0}, \forall n \geq 1$, then $\left\{\tilde{S}_{n}, \mathcal{F}_{n}\right\}$ is a fuzzy martingale.

The above corollary shows the importance of independence in this paper, which is used in the examples.

Remark 2. If $\left\{\tilde{X}_{n}, n \geq 1\right\}$ is a sequence of fuzzy random variables, then $\left\{\tilde{S}_{n}, \mathcal{F}_{n}\right\}$ is not always fuzzy martingale.

Example 1. If $\left\{\tilde{X}_{n}, n \geq 1\right\}$ is a sequence of nonnegative fuzzy random variables, then $\left\{\tilde{S}_{n}, \mathcal{F}_{n}\right\}$ is not a fuzzy martingale, but it is a fuzzy sub-martingale.

Theorem 2. If $\left\{\tilde{S}_{n}, \mathcal{F}_{n}\right\}$ is a fuzzy martingale, then there exists a constant $C$ depending only on p such that

(i) $E|| \tilde{S}_{n}\left\|_{p, q}^{p} \leq C \sum_{i=1}^{n} E\right\| \tilde{X}_{i} \|_{p, q}^{p}, \forall p \in[1,2]$ and $q \in[0,1]$

(ii) $E\left\|\tilde{S}_{n}\right\|_{p, q}^{p} \leq C n^{\frac{p}{2}} \sum_{i=1}^{n} E\left\|\tilde{X}_{i}\right\|_{p, q}^{p} \forall p>2$ and $q \in[0,1]$

where, $\tilde{S}_{n}=\oplus_{i=1}^{n} \tilde{X}_{i}$. 
Proof. We have done the following:

(i) By invoking Fubini's theorem (FT, for short), Burkholder's inequality (BI, for short), and $C_{r}$ inequality $\left(C_{r} \mathrm{I}\right.$, for short), we obtain

$$
\begin{aligned}
E|| \tilde{S}_{n}||_{p, q}^{p} & =E \int_{0}^{1} q\left|\tilde{S}_{n}^{+}(r)\right|^{p}+(1-q)\left|\tilde{S}_{n}^{-}(r)\right|^{p} d r \\
& =\int_{0}^{1} q E\left|\tilde{S}_{n}^{+}(r)\right|^{p}+(1-q) E\left|\tilde{S}_{n}^{-}(r)\right|^{p} d r(\mathrm{FT}) \\
& \leq C \int_{0}^{1} q E\left|\sum_{i=1}^{n}\left[\tilde{X}_{i}^{+}(r)\right]^{2}\right|^{\frac{p}{2}}+(1-q) E\left|\sum_{i=1}^{n}\left[\tilde{X}_{i}^{-}(r)\right]^{2}\right|^{\frac{p}{2}} d r(\mathrm{BI}) \\
& \leq C \sum_{i=1}^{n} \int_{0}^{1} q E\left|\tilde{X}_{i}^{+}(r)\right|^{p}+(1-q) E\left|\tilde{X}_{i}^{-}(r)\right|^{p} d r\left(C_{r} I\right) \\
& =C \sum_{i=1}^{n} E|| \tilde{X}_{i} \|_{p, q}^{p} .
\end{aligned}
$$

(ii) Fubini's theorem (FT), Rosenthal's inequality (RI), $C_{r}$ inequality, and conditional Jensen's inequality (CJI) imply that

$$
\begin{aligned}
& E|| \tilde{S}_{n}||_{p, q}^{p}=E \int_{0}^{1} q\left|\tilde{S}_{n}^{+}(r)\right|^{p}+(1-q)\left|\tilde{S}_{n}^{-}(r)\right|^{p} d r \\
& =\int_{0}^{1} q E\left|\tilde{S}_{n}^{+}(r)\right|^{p}+(1-q) E\left|\tilde{S}_{n}^{-}(r)\right|^{p} d r(\mathrm{FT}) \\
& \leq \int_{0}^{1} q\left\{E\left(\sum_{i=1}^{n} E\left(\tilde{X}_{i}^{+2}(r) \mid \mathcal{F}_{i-1}\right)\right)^{\frac{p}{2}}+\sum_{i=1}^{n} E\left|\tilde{X}_{i}^{+}(r)\right|^{p}\right\} \\
& +(1-q)\left\{E\left(\sum_{i=1}^{n} E\left(\tilde{X}_{i}^{-2}(r) \mid \mathcal{F}_{i-1}\right)\right)^{\frac{p}{2}}+\sum_{i=1}^{n} E\left|\tilde{X}_{i}^{-}(r)\right|^{p}\right\} d r(\mathrm{RI}) \\
& \leq C n^{\frac{p}{2}} \int_{0}^{1} q\left\{\sum_{i=1}^{n} E\left[E\left(\tilde{X}_{i}^{+^{2}}(r) \mid \mathcal{F}_{i-1}\right)\right]^{\frac{p}{2}}+E\left|\tilde{X}_{i}^{+}(r)\right|^{p}\right\} \\
& +(1-q) \sum_{i=1}^{n}\left\{E\left[E\left(\tilde{X}_{i}^{+^{2}}(r) \mid \mathcal{F}_{i-1}\right)\right]^{\frac{p}{2}}+E\left|\tilde{X}_{i}^{+}(r)\right|^{p}\right\} d r\left(C_{r} I\right) \\
& \leq C n^{\frac{p}{2}} \int_{0}^{1} q\left\{\sum_{i=1}^{n} E E\left(\left|\tilde{X}_{i}^{+}(r)\right|^{p} \mid \mathcal{F}_{i-1}\right)+E\left|\tilde{X}_{i}^{+}(r)\right|^{p}\right\} \\
& +(1-q) \sum_{i=1}^{n}\left\{E E\left(\left|\tilde{X}_{i}^{-}(r)\right|^{p} \mid \mathcal{F}_{i-1}\right)+E\left|\tilde{X}_{i}^{-}(r)\right|^{p}\right\} d r(\mathrm{CJI}) \\
& =C n^{\frac{p}{2}} \sum_{i=1}^{n} E \int_{0}^{1} q\left|\tilde{X}_{i}^{+}(r)\right|^{p}+(1-q)\left|\tilde{X}_{i}^{-}(r)\right|^{p} d r(\mathrm{FT}) \\
& =C n^{\frac{p}{2}} \sum_{i=1}^{n} E\left\|\tilde{X}_{i}\right\|_{p, q}^{p}
\end{aligned}
$$

It should be noted that the above theorem is a generalization of Theorem 2.11 of [23] to fuzzy martingales. 
Now we state and prove two theorems which are extensions of Theorem 2.13 in [23] and Theorem 9.5 of [24], respectively.

Theorem 3. If $\left\{\tilde{S}_{n}, \mathcal{F}_{n}\right\}$ is a fuzzy martingale, then there exists a constant $C$ depending only on $p$ such that

$$
E D_{2, q}^{2}\left(\oplus_{i=1}^{n} \tilde{X}_{i}, \oplus_{i=1}^{n} E\left(\tilde{X}_{i} \mid \mathcal{F}_{i-1}\right)\right) \leq C \sum_{i=1}^{n} E D_{2, q}^{2}\left[\tilde{X}_{i}, E\left(\tilde{X}_{i} \mid \mathcal{F}_{i-1}\right)\right], \quad \forall q \in[0,1]
$$

Proof. It is obvious that $\left\{\sum_{i=1}^{n}\left[\tilde{X}_{i}^{+}(r)-E\left(\tilde{X}_{i}^{+}(r) \mid \mathcal{F}_{i-1}\right)\right], \mathcal{F}_{n}\right\}$ and $\left\{\sum_{i=1}^{n}\left[\tilde{X}_{i}^{-}(r)-\right.\right.$ $\left.\left.E\left(\tilde{X}_{i}^{-}(r) \mid \mathcal{F}_{i-1}\right)\right], \mathcal{F}_{n}\right\}$ constitute ordinary martingales. Also, it is easy to see that

$$
E\left[\tilde{X}_{i}^{+}(r)-E\left(\tilde{X}_{i}^{+}(r) \mid \mathcal{F}_{i-1}\right)\right]^{2}=E\left[\tilde{X}_{i}^{+}(r)\right]^{2}-E\left[E\left(\tilde{X}_{i}^{+}(r) \mid \mathcal{F}_{i-1}\right)\right]^{2} \forall r \in[0,1],
$$

and

$$
E\left[\tilde{X}_{i}^{-}(r)-E\left(\tilde{X}_{i}^{-}(r) \mid \mathcal{F}_{i-1}\right)\right]^{2}=E\left[\tilde{X}_{i}^{-}(r)\right]^{2}-E\left[E\left(\tilde{X}_{i}^{-}(r) \mid \mathcal{F}_{i-1}\right)\right]^{2} \forall r \in[0,1]
$$

By invoking Fubini's theorem, Burkholder's inequality, and relations (2) and (3), we obtain

$$
\begin{aligned}
& E D_{2, q}^{2}\left(\tilde{S}_{n}, \oplus_{i=1}^{n} E\left(\tilde{X}_{i}^{+}(r) \mid \mathcal{F}_{i-1}\right)\right) \\
& =E \int_{0}^{1}(1-q)\left(\sum_{i=1}^{n}\left[\tilde{X}_{i}^{+}(r)-E\left(\tilde{X}_{i}^{+} \mid \mathcal{F}_{i-1}\right)\right]\right)^{2}+q\left(\sum_{i=1}^{n}\left[\tilde{X}_{i}^{-}(r)-E\left(\tilde{X}_{i}^{-}(r) \mid \mathcal{F}_{i-1}\right)\right]\right)^{2} d r \\
& =\int_{0}^{1}(1-q) E\left(\sum_{i=1}^{n}\left[\tilde{X}_{i}^{+}(r)-E\left(\tilde{X}_{i}^{+}(r) \mid \mathcal{F}_{i-1}\right)\right]\right)^{2}+q E\left(\sum_{i=1}^{n}\left[\tilde{X}_{i}^{-}(r)-E\left(\tilde{X}_{i}^{-} \mid \mathcal{F}_{i-1}\right)\right]\right)^{2} d r \\
& \leq C \int_{0}^{1}(1-q) \sum_{i=1}^{n} E\left(\left[\tilde{X}_{i}^{+}(r)-E\left(\tilde{X}_{i}^{+}(r) \mid \mathcal{F}_{i-1}\right)\right]\right)^{2}+q \sum_{i=1}^{n} E\left(\left[\tilde{X}_{i}^{-}(r)-E\left(\tilde{X}_{i}^{-}(r) \mid \mathcal{F}_{i-1}\right)\right]\right)^{2} d r \\
& =C \sum_{i=1}^{n} \int_{0}^{1}(1-q)\left\{E\left[\tilde{X}_{i}^{+}(r)\right]^{2}-E\left[E\left(\tilde{X}_{i}^{+}(r) \mid \mathcal{F}_{i-1}\right)\right]^{2}\right\}+q\left\{E\left[\tilde{X}_{i}^{-}(r)\right]^{2}-E\left[E\left(\tilde{X}_{i}^{-}(r) \mid \mathcal{F}_{i-1}\right)\right]^{2}\right\} d r \\
& =C \sum_{i=1}^{n} E \int_{0}^{1}(1-q)\left\{\left[\tilde{X}_{i}^{+}(r)\right]^{2}-\left[E\left(\tilde{X}_{i}^{+}(r) \mid \mathcal{F}_{i-1}\right)\right]^{2}\right\}+q\left\{\left[\tilde{X}_{i}^{-}(r)\right]^{2}-\left[E\left(\tilde{X}_{i}^{-}(r) \mid \mathcal{F}_{i-1}\right)\right]^{2}\right\} d r \\
& =C \sum_{i=1}^{n} E D_{2, q}^{2}\left[\tilde{X}_{i}, E\left(\tilde{X}_{i} \mid \mathcal{F}_{i-1}\right)\right] .
\end{aligned}
$$

Theorem 4. If $\left\{\tilde{S}_{n}, \mathcal{F}_{n}\right\}$ is a fuzzy martingale and $1 \leq p \leq 2$, then

$$
E \max _{1 \leq k \leq n}\left\|\tilde{S}_{k}\right\|_{p, q}^{p} \leq C \sum_{i=1}^{n} E\left\|\tilde{X}_{i}\right\|_{p, q}^{p}
$$


Proof. By using monotonicity property of integral (MPI, for short), Fubini's theorem, Burkholder's inequality, and $C_{r}$ inequality, we obtain

$$
\begin{aligned}
E \max _{1 \leq k \leq n} \| \tilde{S}_{k}||_{p, q}^{p} & =E \max _{1 \leq k \leq n} \int_{0}^{1} q\left|\tilde{S}_{k}^{+}(r)\right|^{p}+(1-q)\left|\tilde{S}_{k}^{-}(r)\right|^{p} d r \\
& \leq E \int_{0}^{1}\left\{q \max _{1 \leq k \leq n}\left|\tilde{S}_{k}^{+}(r)\right|^{p}+(1-q) \max _{1 \leq k \leq n}\left|\tilde{S}_{k}\right|^{p}\right\} d r(\mathrm{MPI}) \\
& =\int_{0}^{1}\left\{q E \max _{1 \leq k \leq n}\left|\tilde{S}_{k}^{+}(r)\right|^{p}+(1-q) E \max _{1 \leq k \leq n}\left|\tilde{S}_{k}^{-}(r)\right|^{p}\right\} d r(\mathrm{FT}) \\
& \leq C \int_{0}^{1} q E\left(\sum_{i=1}^{n}\left(\tilde{X}_{i}^{+}(r)\right)^{2}\right)^{\frac{p}{2}}+(1-q) E\left(\sum_{i=1}^{n}\left(\tilde{X}_{i}^{-}(r)\right)^{2}\right)^{\frac{p}{2}} d r \\
& =C \int_{0}^{1}\left\{q \sum_{i=1}^{n} E\left|\tilde{X}_{i}^{+}(r)\right|^{p}+(1-q) \sum_{i=1}^{n} E\left|\tilde{X}_{i}^{-}(r)\right|^{p}\right\} d r\left(C_{r} I\right) \\
& =C \sum_{i=1}^{n} E\left\|\tilde{X}_{i}\right\|_{p, q}^{p} .
\end{aligned}
$$

\section{Convergence Theorems}

In this section, by invoking theorems of previous section, we establish some weak and strong convergence theorems for fuzzy martingales.

Theorem 5. If $\left\{\tilde{X}_{n}, \mathcal{F}_{n}\right\}$ is a fuzzy martingale, then

$$
E d_{*}^{2}\left(\tilde{X}_{n}, \tilde{X}_{m}\right)=E\left\|\tilde{X}_{n}\right\|_{*}^{2}-E\left\|\tilde{X}_{m}\right\|_{*}^{2}, \text { for } n>m
$$

Proof. By invoking Fubini's theorem and orthogonality lemma of ordinary martingale (OLOM, for short), we obtain

$$
\begin{aligned}
E d_{*}^{2}\left(\tilde{X}_{n}, \tilde{X}_{m}\right) & =E \int_{0}^{1}\left\{\left|\tilde{X}_{n}^{-}(r)-\tilde{X}_{m}^{-}(r)\right|^{2}+\left|\tilde{X}_{n}^{+}(r)-\tilde{X}_{m}^{+}(r)\right|^{2}\right\} d r \\
& =\int_{0}^{1}\left\{E\left|\tilde{X}_{n}^{-}(r)-\tilde{X}_{m}^{-}(r)\right|^{2}+E\left|\tilde{X}_{n}^{+}(r)-\tilde{X}_{m}^{+}(r)\right|^{2}\right\} d r \text { (FT) } \\
& =\int_{0}^{1}\left\{E\left|\tilde{X}_{n}^{-}(r)\right|^{2}-E\left|\tilde{X}_{m}^{-}(r)\right|^{2}+E\left|\tilde{X}_{n}^{+}(r)\right|^{2}-E\left|\tilde{X}_{m}^{+}(r)\right|^{2}\right\} d r \text { (OLOM) } \\
& =E|| \tilde{X}_{n}\left\|_{*}^{2}-E|| \tilde{X}_{m}\right\|_{*}^{2} .
\end{aligned}
$$

Note that Theorem 5 is a generalization of Lemma 10.4.1 of [24] to fuzzy martingales.

Corollary 2. If $\left\{\tilde{X}_{n}, n \geq 1\right\}$ is a fuzzy martingale such that $\sup _{n \geq 1} E\left\|\tilde{X}_{n}\right\|_{*}^{2}<\infty$, then $\left\{\tilde{X}_{n}, n \geq 1\right\}$ is Cauchy convergent in $L^{2}$ with respect to the metric $d_{*}$.

Lemma 2. If $\left\{\tilde{X}_{n}, \mathcal{F}_{n}\right\}$ is a sequence of FRVs, then

$$
\left\|E\left(\tilde{X}_{n+1} \mid \mathcal{F}_{n}\right)\right\|_{*}^{2} \leq E\left(\left\|\tilde{X}_{n+1}\right\|_{*}^{2} \mid \mathcal{F}_{n}\right)
$$


Proof. By using conditional Jensen's inequality and Fubini's theorem, we obtain

$$
\begin{aligned}
\left\|E\left(\tilde{X}_{n+1} \mid \mathcal{F}_{n}\right)\right\|_{*}^{2} & =\int_{0}^{1}\left\{E^{2}\left(\tilde{X}_{n+1}^{+}(r) \mid \mathcal{F}_{n}\right)+E^{2}\left(\tilde{X}_{n+1}^{-}(r) \mid \mathcal{F}_{n}\right)\right\} d r \\
& \leq \int_{0}^{1}\left\{E\left(\left\{\tilde{X}_{n+1}^{+}(r)\right\}^{2} \mid \mathcal{F}_{n}\right)+E\left(\left\{\tilde{X}_{n+1}^{-}(r)\right\}^{2} \mid \mathcal{F}_{n}\right)\right\} d r(\mathrm{CJI}) \\
& =E\left(|| \tilde{X}_{n+1}||_{*}^{2} \mid \mathcal{F}_{n}\right)(\mathrm{FT}) .
\end{aligned}
$$

Corollary 3. If $\left\{\tilde{X}_{n}, n \geq 1\right\}$ is a fuzzy martingale, then $\left\{\left\|\tilde{X}_{n}\right\|_{*}^{2}, n \geq 1\right\}$ is a real-valued sub-martingale.

Theorem 6. Let $\left\{\tilde{S}_{n}, \mathcal{F}_{n}\right\}$ be a fuzzy martingale and $p>2$, then $\frac{\tilde{S}_{n}}{n} \rightarrow \tilde{0}$ in probability with respect to the metric $D_{p, q}$ if

$$
\sum_{i=1}^{n} E\left\|\tilde{X}_{i}\right\|_{p, q}^{p}=o\left(n^{\frac{p}{2}}\right) \text { as } n \rightarrow \infty .
$$

Proof. By using Markov's inequality and Theorem 2, the proof is straightforward.

Example 2. Let $\left\{\tilde{X}_{n}, n \geq 1\right\}$ be a sequence of independent fuzzy random variables with the following membership functions:

$$
\mu_{\tilde{X}_{n}(\omega)}(x)= \begin{cases}\frac{x-X_{n}(\omega)}{X_{n}(\omega)}, & X_{n}(\omega)<x \leq 2 X_{n}(\omega), \\ \frac{3 X_{n}(\omega)-x}{X_{n}(\omega)}, & 2 X_{n}(\omega)<x<3 X_{n}(\omega), \\ 0, & \text { otherwise, }\end{cases}
$$

where $\left\{X_{n}, n \geq 1\right\}$ is a sequence of independent random variables with the following probability $P\left(X_{n}=n^{\beta}\right)=\frac{1}{2}=P\left(X_{n}=-n^{\beta}\right)$.

It is easy to see that

$$
\left\|\tilde{X}_{n}\right\|_{p, q}^{p}=W_{p, q}\left|X_{n}\right|^{p}
$$

where

$$
W_{p, q}=\frac{q}{p+1}\left[2^{p+1}-1\right]-\frac{1-q}{p+1}\left[2^{p+1}-3^{p+1}\right] .
$$

We want to prove that $\sum_{i=1}^{n} E|| \tilde{X}_{i} \|_{p, q}^{p}=o\left(n^{\frac{p}{2}}\right)$, i.e., $\frac{1}{n^{\frac{p}{2}}} \sum_{i=1}^{n} i^{\beta p} \rightarrow 0$ as $n \rightarrow \infty$. By using Kronecker's lemma, it suffices to show that

$$
\sum_{n=1}^{\infty} \frac{1}{n^{p\left(\frac{1}{2}-\beta\right)}}<\infty
$$

If $p>\frac{2}{1-2 \beta}$, then

$$
\sum_{n=1}^{\infty} \frac{1}{n^{p\left(\frac{1}{2}-\beta\right)}}<\infty
$$

By using Theorem 6, we obtain $\frac{\tilde{S}_{n}}{n} \rightarrow \tilde{0}$ in probability with respect to the metric $D_{p, q}$ as $n \rightarrow \infty$. 
The following theorem is an extension of Theorem 2.13 in [23] to fuzzy martingales.

Theorem 7. Let $\left\{\tilde{S}_{n}, n \geq 1\right\}$ be a martingale and $\left\{b_{n}\right\}$ be a sequence of positive constants with $b_{n} \uparrow \infty$. Then $b_{n}^{-1} \tilde{S}_{n} \rightarrow \tilde{0}$ in probability with respect to the metric $D_{2, q}$, if

$$
\begin{aligned}
& \sum_{i=1}^{n} P\left(\left\|\tilde{X}_{i}\right\|_{2, q}>b_{n}\right) \rightarrow 0 \\
& b_{n}^{-1} \sum_{i=1}^{n}\left\|E\left(\tilde{X}_{n i} \mid \mathcal{F}_{i-1}\right)\right\|_{2, q} \rightarrow 0 \text { in probability, } \\
& b_{n}^{-2} \sum_{i=1}^{n}\left\{E D_{2, q}^{2}\left[\tilde{X}_{n, i}, E\left(\tilde{X}_{n, i} \mid \mathcal{F}_{i-1}\right)\right]\right\} \rightarrow 0
\end{aligned}
$$

where, $\tilde{X}_{n i}=\tilde{X}_{i} I_{\left\{|| \tilde{X}_{i} \|_{2, q} \leq n\right\}}$ and $\tilde{S}_{n}=\bigoplus_{i=1}^{n} \tilde{X}_{i}$.

Proof. Suppose that $\tilde{S}_{n n}=\bigoplus_{i=1}^{n} \tilde{X}_{n, i}$. It is easy to see that

$$
b_{n}^{-1}|| \tilde{S}_{n}\left\|_{2, q} \leq b_{n}^{-1} D_{2, q}\left(\tilde{S}_{n}, \tilde{S}_{n n}\right)+b_{n}^{-1}\right\| \tilde{S}_{n n} \|_{2, q} .
$$

We want to prove $b_{n}^{-1}|| \tilde{S}_{n} \|_{2, q} \rightarrow 0$ in probability. By using relation (7), we should prove that (a) $b_{n}^{-1} D_{2, q}\left(\tilde{S}_{n}, \tilde{S}_{n n}\right) \rightarrow 0$ and (b) $b_{n}^{-1}|| \tilde{S}_{n n} \|_{2, q} \rightarrow 0$ in probability:

(a) For each $\epsilon>0$, we have

$$
\begin{aligned}
P\left(b_{n}^{-1} D_{2, q}\left(\tilde{S}_{n}, \tilde{S}_{n n}\right)>\epsilon\right) & =P\left(\tilde{S}_{n} \neq \tilde{S}_{n n}\right) \\
& \leq \sum_{i=1}^{n} P\left(\left\|\tilde{X}_{i}\right\|_{2, q}>b_{n}\right) \rightarrow 0 .
\end{aligned}
$$

Thus, $b_{n}^{-1} D_{2, q}\left(\tilde{S}_{n}, \tilde{S}_{n n}\right) \rightarrow 0$ in probability.

(b) By using sub-additivity of the metric $D_{2, q}$, we obtain

$$
b_{n}^{-1}|| \tilde{S}_{n n}\left\|_{2, q} \leq b_{n}^{-1} D_{2, q}\left(\tilde{S}_{n n}, \oplus_{i=1}^{n} E\left(\tilde{X}_{n i} \mid \mathcal{F}_{i-1}\right)\right)+\right\| \oplus_{i=1}^{n} E\left(\tilde{X}_{n, i} \mid \mathcal{F}_{i-1}\right) \|_{2, q} .
$$

The sub-additivity of the norm $\|\cdot\|_{2, q}$ and condition (3) imply that $b_{n}^{-1} \| \oplus_{i=1}^{n}$ $E\left(\tilde{X}_{n i} \mid \mathcal{F}_{i-1}\right)\left\|_{2, q} \leq b_{n}^{-1} \sum_{i=1}^{n}\right\| E\left(\tilde{X}_{n i} \mid \mathcal{F}_{i-1}\right) \|_{2, q} \rightarrow 0$ in probability.

It remains to show that $b_{n}^{-1} D_{2, q}\left(\tilde{S}_{n n}, \oplus_{i=1}^{n} E\left(\tilde{X}_{n i} \mid \mathcal{F}_{i-1}\right)\right) \rightarrow 0$ in probability.

By using Markov's inequality and Theorem 3, we have

$$
\begin{aligned}
P\left(b_{n}^{-1} D_{2, q}\left(\tilde{S}_{n n}, \oplus_{i=1}^{n} E\left(\tilde{X}_{n i} \mid \mathcal{F}_{i-1}\right)\right)>\epsilon\right) & \leq \frac{E D_{2, q}^{2}\left(\tilde{S}_{n n}, \oplus_{i=1}^{n} E\left(\tilde{X}_{n i} \mid \mathcal{F}_{i-1}\right)\right)}{b_{n}^{2} \epsilon^{2}} \\
& \leq \frac{C \sum_{i=1}^{n} E D_{2, q}^{2}\left(\tilde{X}_{n i}, E\left(\tilde{X}_{n i} \mid \mathcal{F}_{i-1}\right)\right)}{b_{n}^{2} \epsilon^{2}} \rightarrow 0 .
\end{aligned}
$$

This completes the proof.

The following example is an evidence of Theorem 7.

Example 3. Suppose that $\left\{\tilde{X}_{n}, n \geq 1\right\}$ is a sequence of independent FRVs with the following probability function:

$$
P\left(\tilde{X}_{n}=n^{-\alpha} \tilde{u}\right)=P\left(\tilde{X}_{n}=-n^{-\alpha} \tilde{u}\right)=\frac{1}{2},
$$


where $\tilde{u}$ is a fuzzy number such that $\|\tilde{u}\|_{p, q}=1$. It is obvious that

(i) $\sum_{i=1}^{n} P\left(\left\|\tilde{X}_{i}\right\|_{2, q}>n\right)=0$

We want to show that

(ii) $n^{-1} \sum_{i=1}^{n}\left\|E\left(\tilde{X}_{n i} \mid \mathcal{F}_{i-1}\right)\right\|_{2, q} \rightarrow 0$ in probability.

By invoking Markov's inequality and Lemma 2, we obtain

$$
\begin{aligned}
P\left(n^{-1} \sum_{i=1}^{n}\left\|E\left(\tilde{X}_{n i} \mid \mathcal{F}_{i-1}\right)\right\|_{2, q}>\epsilon\right) & \leq \frac{\sum_{i=1}^{n} E|| E\left(\tilde{X}_{n i} \mid \mathcal{F}_{i-1}\right) \|_{2, q}}{n \epsilon} \\
& \leq \frac{\sum_{i=1}^{n} E E^{\frac{1}{2}}\left(\left\|\tilde{X}_{n i}\right\|_{2, q}^{2} \mid \mathcal{F}_{i-1}\right)}{n \epsilon} \\
& =\frac{1}{n \epsilon} \sum_{i=1}^{n} \frac{1}{i^{\alpha}}
\end{aligned}
$$

By using Kronecker's lemma $\frac{1}{n} \sum_{i=1}^{n} \frac{1}{i^{\alpha}} \rightarrow 0$, and consequently

$$
n^{-1} \sum_{i=1}^{n} E\left(\tilde{X}_{n i} \mid \mathcal{F}_{i-1}\right) \rightarrow 0 \text { in probability. }
$$

It remains to show that

(iii) $n^{-2} \sum_{i=1}^{n} E D_{2, q}^{2}\left(\tilde{X}_{n i}, E\left(\tilde{X}_{n i} \mid \mathcal{F}_{i-1}\right)\right) \rightarrow 0$ as $n \rightarrow \infty$.

It is easy to see that

$$
n^{-2} \sum_{i=1}^{n} E D_{2, q}^{2}\left(\tilde{X}_{n i}, E\left(\tilde{X}_{n i} \mid \mathcal{F}_{i-1}\right)\right)=n^{-2} \sum_{i=1}^{n} \frac{1}{i^{2 \alpha}}
$$

By invoking Kronecker's lemma, we obtain $n^{-2} \sum_{i=1}^{n} \frac{1}{i^{2 \alpha}} \rightarrow 0$ and thus,

$$
n^{-2} \sum_{i=1}^{n} E D_{2, q}^{2}\left(\tilde{X}_{n i}, E\left(\tilde{X}_{n i} \mid \mathcal{F}_{i-1}\right)\right) \rightarrow 0 \text { as } n \rightarrow \infty
$$

Therefore, all of the conditions of the above theorem hold, thus $\frac{\tilde{S}_{n}}{n} \rightarrow \tilde{0}$ in probability with respect to the metric $D_{2, q}$.

Theorem 8. Let $\left\{\tilde{S}_{n}, \mathcal{F}_{n}\right\}$ be a fuzzy martingale. If for all $1 \leq p<2,\left\{\left\|\tilde{X}_{n}\right\|_{p, q}^{p}, n \geq 1\right\}$ is uniformly integrable, then $n^{-2} E|| \tilde{S}_{n} \|_{p, q}^{p} \rightarrow 0$ as $n \rightarrow \infty$.

Proof. By invoking Theorem 2, we obtain

$$
E|| \tilde{S}_{n}\left\|_{p, q}^{p} \leq C \sum_{i=1}^{n} E\right\| \tilde{X}_{i} \|_{p, q}^{p} .
$$


Since $\left\{\left\|\tilde{X}_{n}\right\|_{p, q}^{p}, n \geq 1\right\}$ is uniformly integrable, by invoking Theorem 5.4.1 in [24] $\sup _{n \geq 1} E|| \tilde{X}_{n} \|_{p, q}^{p}<\infty$ i.e., $\exists M$ s.t. $\sup _{n \geq 1} E\left\|\tilde{X}_{n}\right\|_{p, q}^{p}<M$. Hence,

$$
\begin{aligned}
E\left\|\tilde{S}_{n}\right\|_{p, q}^{p} & \leq C \sum_{i=1}^{n} E|| \tilde{X}_{i} \|_{p, q}^{p} \\
& \leq C n \sup _{n \geq 1} E|| \tilde{X}_{n} \|_{p, q}^{p} \\
& \leq C n M .
\end{aligned}
$$

So,

$$
n^{-2} E\left\|\tilde{S}_{n}\right\|_{p, q}^{p} \leq C n^{-1} M
$$

which implies that $n^{-2} E|| \tilde{S}_{n} \|_{p, q}^{p} \rightarrow 0$.

The above theorem is a generalization of Theorem 2.22 in [23] to fuzzy martingales.

Example 4. Suppose that $\left\{\tilde{X}_{n}, n \geq 1\right\}$ is a sequence of independent Fs with the following probability function:

$$
P\left(\tilde{X}_{n}=n^{-1} \tilde{u}\right)=\frac{1}{n}, P\left(\tilde{X}_{n}=\frac{-\tilde{u}}{n(n-1)}\right)=1-\frac{1}{n},
$$

where $\tilde{u}$ is a fuzzy number with the following membership function:

$$
\tilde{u}(x)=1-\frac{\sqrt{6}}{3}|x|, \quad-\frac{\sqrt{6}}{2} \leq x \leq \frac{\sqrt{6}}{2} .
$$

It is easy to see that $\left\|\tilde{X}_{n}\right\|_{p, q}^{p}$ is uniformly bounded and, consequently, is uniformly integrable. Thus, by invoking Theorem $8, n^{-2} E|| \tilde{S}_{n} \|_{p, q}^{p} \rightarrow 0$ as $n \rightarrow \infty$.

Theorem 9. If $\left\{\tilde{S}_{n}, \mathcal{F}_{n}\right\}$ is a fuzzy martingale such that $\sum_{n=1}^{\infty} E\left\|\tilde{X}_{n}\right\|_{p, q}^{p}<\infty$ for all $p \in$ $[1,2]$, then $\oplus_{n=1}^{\infty} \tilde{X}_{n}$ converges almost surely with respect to the metric $D_{p, q}$. In particular case, $\oplus_{n=1}^{\infty} \tilde{X}_{n}$ converges almost surely with respect to the metric $d_{*}$ if $\sum_{n=1}^{\infty} \operatorname{Var}\left(\tilde{X}_{n}\right)<\infty$.

Proof. The completeness of the metric space $\left(E, D_{p, q}\right)$ implies that $\oplus_{n=1}^{\infty} \tilde{X}_{n}$ converges almost surely with respect to the metric $D_{p, q}$ iff $\sup _{k \geq n} D_{p, q}\left(\tilde{S}_{k}, \tilde{S}_{n}\right) \rightarrow 0$ in probability as $n \rightarrow \infty$. Let $n<m$, and consider a Cauchy sequence. Theorem 4 implies that

$$
\begin{aligned}
P\left(\max _{n \leq k \leq m} D_{p, q}\left(\tilde{S}_{k}, \tilde{S}_{n}\right)>\epsilon\right) & \leq \frac{E \max _{n \leq k \leq m} D_{p, q}^{p}\left(\tilde{S}_{k}, \tilde{S}_{n}\right)}{\epsilon^{p}} \\
& =\frac{E \max _{n \leq k \leq m}\left\|\oplus_{i=n+1}^{k} \tilde{X}_{i}\right\|_{p, q}^{p}}{\epsilon^{p}} \\
& \leq C \sum_{i=n+1}^{m} E\left\|\tilde{X}_{i}\right\|_{p, q}^{p} .
\end{aligned}
$$

Now, letting $m \rightarrow \infty$, we have

$$
P\left(\sup _{k \geq n} D_{p, q}\left(\tilde{S}_{k}, \tilde{S}_{n}\right)>\epsilon\right) \leq \frac{C \sum_{k=n+1}^{\infty} E\left\|\tilde{X}_{k}\right\|_{p, q}^{p}}{\epsilon^{p}} \rightarrow 0 \text { as } n \rightarrow \infty .
$$


Example 5. Let $\left\{\tilde{X}_{n}, n \geq 1\right\}$ be a sequence of independent fuzzy random variables with the following membership functions:

$$
\mu_{\tilde{X}_{n}(\omega)}(x)= \begin{cases}\frac{x-X_{n}(\omega)}{X_{n}(\omega)}, & X_{n}(\omega)<x \leq 2 X_{n}(\omega), \\ \frac{3 X_{n}(\omega)-x}{X_{n}(\omega)}, & 2 X_{n}(\omega)<x<3 X_{n}(\omega), \\ 0, & \text { otherwise, }\end{cases}
$$

where $\left\{X_{n}, n \geq 1\right\}$ is a sequence of independent random variables such that $\operatorname{Var}\left(X_{n}\right)=$ $n^{\beta} \sigma^{2}$. By considering $p=2$ and $q=\frac{1}{2}$, we have $E D_{p, q}^{p}\left(\tilde{X}_{n}, E \tilde{X}_{n}\right)=\operatorname{Var}\left(\tilde{X}_{n}\right)$. For establishing a strong convergence of $\left\{\tilde{S}_{n}, n \geq 1\right\}$ with respect to the metric $D_{p, q}$, it remains to show that $\sum_{n=1}^{\infty} \operatorname{Var}\left(\tilde{X}_{n}\right)<\infty$. It is easy to see that $\operatorname{Var}\left(\tilde{X}_{n}\right)=\frac{19}{6} n^{\beta} \sigma^{2}$, and $\sum_{n=1}^{\infty} \operatorname{Var}\left(\tilde{X}_{n}\right)=$ $\frac{19 \sigma^{2}}{6} \sum_{n=1}^{\infty} n^{\beta}<\infty$, where $\beta<-1$. Therefore, Theorem 9 implies that $\oplus_{n=1}^{\infty} \tilde{X}_{n}$ converges almost surely with respect to the metric $D_{p, q}$.

\section{Conclusions}

In this article, we extended some well-known inequalities to fuzzy martingales based on the metric $D_{p, q}$. Also, by invoking these inequalities, we established some limit theorems for fuzzy martingales. It is mentioned that if fuzzy random variables reduce to ordinary (real-valued) random variables, all results hold. The study of weak convergence and strong convergence of fuzzy sub-(super-) martingales is a potential topic for future research.

\section{Acknowledgements}

The authors are grateful to referees for their useful and constructive suggestions which lead to an improved version of the paper. The third author would like to thank University of Tehran for partial support of this research.

\section{Author details}

${ }^{1}$ Department of Statistics, Faculty of Mathematical Sciences, Ferdowsi University of Mashhad, Mashhad 91775-1159, Iran.

${ }^{2}$ Department of Engineering Science, College of Engineering, University of Tehran, Tehran 11365-4563, Iran.

Received: 1 August 2013 Accepted: 26 February 2014

Published: 31 March 2014

\section{References}

1. Kwakernaak, H: Fuzzy random variables I. Inform. Sci. 15, 1-29 (1978)

2. Miyakoshi, M, Shimbo, M: A strong law of large numbers for fuzzy random variables. Fuzzy Set. Syst. 12, 133-142 (1984)

3. Puri, ML, Ralescu, DA: Fuzzy random variables. J. Math. Anal. Appl. 114, 402-422 (1986)

4. Puri, ML, Ralescu, DA: Convergence theorems for fuzzy martingales. J. Math. Anal. Appl. 160, 107-122 (1991)

5. Miranda, E, Couso, I, Gil, P: Random sets as imprecise random variables. J. Math. Anal. Appl. 307, 32-47 (2005)

6. Klement, EP, Puri, ML, Ralescu, DA: Limit theorems for fuzzy random variables. Proc. Roy. Soc. London Ser. A. 407, 171-182 (1986)

7. Taylor, RL, Seymour, L, Chen, Y: Weak laws of large numbers for fuzzy random sets. Nonlinear Anal. 47, 114-125 (2001)

8. Joo, SY, Kim, YK, Kwon, JS: On Chung's type law of large numbers for fuzzy random variables. Stat. Probab. Lett. 74, 67-75 (2005)

9. Fu, K, Zhang, L-X: Strong limit theorems for random sets and fuzzy random sets with slowly varying weights. Inform. Sci. 178, 2648-2660 (2008)

10. Korner, R: On the variance of fuzzy random variables. Fuzzy Set. Syst. 92, 83-93 (1997)

11. Fei, W, Wu, R, Shao, S: Doob's stopping theorem for fuzzy (super, sub) martingales with discrete time. Fuzzy Set. Syst. 135, 377-390 (2003)

12. Ralescu, D: Fuzzy random variables revisited. In: Mathematics of Fuzzy Sets, Handbook Fuzzy Sets Series, vol. 3, pp. 701-710, Kluwer, Boston, (1999)

13. Stojakovic, M: Fuzzy martingales-a simple form of fuzzy processes. Stochastic Anal. Appl. 14, 355-367 (1996)

14. Feng, $Y, H u, L, S h u, H$ : The variance and covariance of fuzzy random variables and their applications. Fuzzy Set. Syst. 120, 487-497 (2001)

15. Feng, Y: Convergence theorems for fuzzy random variables and fuzzy martingales. Fuzzy Set. Syst. 103, 435-441 (1999)

16. Feng, Y: An approach to generalize laws of large numbers for fuzzy random variables. Fuzzy Set. Syst. 128, 237-245 (2002)

17. Moore, RE, Kearfott, RB, Cloud, MJ: Introduction to Interval Analysis, Siam, Philadelphia (2009)

18. Viertl, R: Statistical Methods for Fuzzy Data. Wiley, Chichester, West Sussex (2011) 
19. Sadeghpour Gildeh, B, Rahimpour, S: A fuzzy bootstrap test for the mean with $D_{p, q}$-distance. Fuzzy Inf. Eng. 4, 351-358 (2011)

20. Wu, HC: The laws of large numbers for fuzzy random variables. Fuzzy Set. Syst. 116, 245-262 (2000)

21. Aumann, RJ: Integrals of set-valued functions. J. Math. Anal. Appl. 12, 1-12 (1965)

22. Fei, W: Regularity and stopping theorem for fuzzy martingales with continuous parameters. Inform. Sci. 169, 175-187 (2005)

23. Hall, P, Heyde, CC: Martingale Limit Theory and Its Application. Academic, New York (1980)

24. Gut, A: Probability: a Graduate Course. Springer, New York (2005)

doi:10.1186/2195-5468-2-7

Cite this article as: Ahmadzade et al:: Some moment inequalities for fuzzy martingales and their applications. Journal of Uncertainty Analysis and Applications 2014 2:7.

Submit your manuscript to a SpringerOpen ${ }^{\circ}$ journal and benefit from:

- Convenient online submission

- Rigorous peer review

- Immediate publication on acceptance

- Open access: articles freely available online

- High visibility within the field

- Retaining the copyright to your article

Submit your next manuscript at $\gg$ springeropen.com 